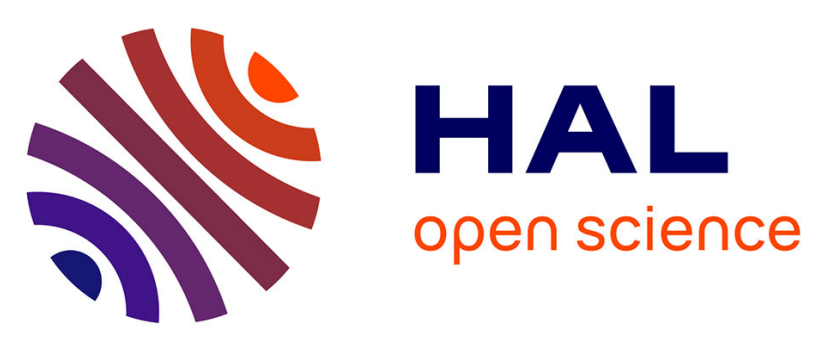

\title{
Relaxation Effects due to Tunnelling of Hydrogen in Metals and Semiconductors
}

G. Cannelli, R. Cantelli, F. Cordero, F. Trequattrini

\section{To cite this version:}

G. Cannelli, R. Cantelli, F. Cordero, F. Trequattrini. Relaxation Effects due to Tunnelling of Hydrogen in Metals and Semiconductors. Journal de Physique IV Proceedings, 1996, 06 (C8), pp.C8-13-C8-26. 10.1051/jp4:1996802 . jpa-00254519

\section{HAL Id: jpa-00254519 https://hal.science/jpa-00254519}

Submitted on 1 Jan 1996

HAL is a multi-disciplinary open access archive for the deposit and dissemination of scientific research documents, whether they are published or not. The documents may come from teaching and research institutions in France or abroad, or from public or private research centers.
L'archive ouverte pluridisciplinaire HAL, est destinée au dépôt et à la diffusion de documents scientifiques de niveau recherche, publiés ou non, émanant des établissements d'enseignement et de recherche français ou étrangers, des laboratoires publics ou privés. 


\title{
Relaxation Effects due to Tunnelling of Hydrogen in Metals and Semiconductors
}

\author{
G. Cannelli, R. Cantelli*, F. Cordero** and F. Trequattrini* \\ Dipartimento di Fisica, Università della Calabria, Arcavacata di Rende (CS), 87036 Italy, and INFM \\ * Dipartimento di Fisica, Università di Roma "La Sapienza", P.le A. Moro 2, 00185 Roma, Italy, and \\ INFM \\ ** CNR, Istituto di Acustica "O.M. Corbino", 00189 Roma, Italy, and INFM
}

\begin{abstract}
The mobility of hydrogen and its isotopes in metals has been the object of investigation for several years, whereas the diffusion studies of $\mathrm{H}$ in doped semiconductors started more recently. Although the $\mathrm{H}$ diffusion coefficient in metals may be several orders of magnitudes higher than in semiconductors, the dynamics of $\mathrm{H}$ in metals and semiconductors presents many common features, like precipitation, trapping by heavier impurities and, as indicated by recent results, quantum tunnelling at low temperature.

In metals two regimes of the $\mathrm{H}$ mobility are observed: hopping with deviations from a classical Arrhenius motion, and a much faster tunnelling within few close sites. In the latter regime the $H$ dynamics does not consist of jumps but of transitions between the quantized energy levels of the tunnel systems. The types of interactions assisting the $\mathrm{H}$ transitions and the geometry of the tunnel systems are an open problem: although the two-level tunnel system (TLS) has been widely used to explain neutron diffusion, specific heat, and acoustic spectroscopy results in interstitial solutions $\left(\mathrm{NbO}_{\mathbf{x}} \mathrm{H}_{\mathrm{y}}\right)$, recently this model has appeared not to be valid in substitutional solutions $\left(\mathrm{NbZr}_{\mathbf{x}} \mathrm{H}_{\mathbf{y}}, \mathrm{Nb}_{\mathrm{x}} \mathrm{Ti}_{\mathbf{y}}\right)$ where the tunnel systems have a higher symmetry. The four-level systems seem to be more appropriate, although the corresponding model has not been developed as much as the TLS yet.

In boron doped silicon, the relaxation rates $\tau^{-1}(T)$ of $\mathrm{H}$ around $\mathrm{B}$ obtained from anelastic relaxation were joined with those from infrared absorption: the remarkably wide range obtained (11 decades) clearly shows a deviation of $\tau^{-1}(T)$ from the classical dependence at low temperature. However, no conclusions can be drawn at present on the mechanism governing the $H(D)$ dynamics.

Most recently, the investigation of the dynamics of $\mathrm{H}(\mathrm{D})$ in GaAs doped with $\mathrm{Zn}$ revealed a dissipation peak at $20 \mathrm{~K}$ in the $\mathrm{kHz}$ range. This relaxation has the highest rate found so far for $\mathrm{H}$ in a semiconductor: more than 15 orders of magnitude higher than in all the other semiconductors measured so far. The analysis of the dissipation curves indicates that the nature of the $\mathrm{H}$ reorientation is strongly quantistic.
\end{abstract}

\section{INTRODUCTION}

The surface of metals acts as a cathalizer and splits the $\mathrm{H}_{2}$ molecule, making it possible for $\mathrm{H}$ to enter into the bulk from the gas phase. Thus hydrogen goes spontaneously into solid solution in atomic or protonic form. Although the surface barrier may be remarkably high, varying from metal to metal, the $\mathrm{H}$ bulk mobility is generally rather fast.

The description of the dynamics of interstitial $\mathrm{H}$ needs a fully quantum mechanical treatment. Even its long-range diffusion via overbarrier hopping follows the Arrhenius law only approximately and depends on the isotope mass in a completely non-classical way. Under certain circumstances, $\mathrm{H}$ can perform coherent tunnelling between two or more sites, but band-like long-range delocalization was 
never observed, even for the much lighter positive muon. Hydrogen and its isotopes may have different regimes even within a same $\mathrm{H}$-defect complex. In the $\mathrm{NbO}_{\mathbf{x}} \mathrm{H}_{\mathrm{y}}$ interstitial solution, the most investigated system, a deviation of the $\mathrm{H}-\mathrm{O}$ relaxation rate at the liquid nitrogen temperatures indicated hopping via phonon-assisted incoherent tunnelling of $H$ [1]. In the same system, the extension to liquid helium temperatures of specific heat [2,3] neutron scattering [4-6], and anelastic relaxation measurements in the $\mathrm{MHz}[7,8]$ and $\mathrm{kHz}$ [9-11] range, showed coherent tunnelling of $\mathrm{H}$ around $\mathrm{O}$. In this system and in the other interstitial solutions investigated, $\mathrm{H}$ is thought to be confined within two nearly-equivalent sites, forming a two-level tunnel system (TLS); the TLS model was first developed to explain the low temperature thermal and acoustic properties of glasses [12]. Tunnelling over more than two sites had also been considered for $\mathrm{NbO}_{x} \mathrm{H}_{y}$ [8]; however, this configuration was then abandoned, both because of the successful explanation in terms of TLS, and because there are no more than two equivalent positions for $\mathrm{H}$ near an $\mathrm{O}$ atom. In fact, the formation of a tunnel system over non-equivalent sites in the strongly distorted environment of an $\mathrm{O}$ atom is considered unlikely.

The dynamics of $\mathrm{H}$ in a substitutional solution has been much less studied, although the symmetry of the substitutional-H pair is much simpler. In $\mathrm{NbTi}_{\mathbf{x}} \mathrm{H}_{\mathbf{y}}$ and $\mathrm{NbZr}_{\mathbf{x}} \mathrm{H}_{\mathbf{y}}, \mathrm{H}$ could form a four-level tunnel system (FLS) within the four equivalent tetrahedral sites of one face of the bcc cubic cell containing the substitutional impurity. Indeed, this model has been proposed and found consistent with anelastic relaxation measurements in $\mathrm{NbTi}_{\mathrm{x}} \mathrm{H}_{\mathrm{y}}$ [13]. Also the excess specific heat measured in $\mathrm{Nb}_{0.95} \mathrm{Ti}_{0,05}(\mathrm{H}, \mathrm{D})_{\mathrm{x}}$ down to $50 \mathrm{mK}$ [14] can be explained by supposing that $\mathrm{H}$ forms a FLS. A more stringent evidence of the existence of FLS's was given only recently [15] by acoustic spectroscopy experiments in single crystals of $\mathrm{NbZr}_{\mathrm{x}} \mathrm{H}_{\mathrm{y}}$, exciting vibrations with different symmetries.

In semiconductors, the surface does not split the $\mathrm{H}_{2}$ molecule, therefore the $\mathrm{H}$ charge is usually carried out by ion-gun irradiation of the sample surface, or by plasma exposure. The diffusion coefficient of hydrogen and its isotopes in semiconductors may be several orders of magnitude lower than in metals; nevertheless this light particle can display, locally, a remarkably high mobility. In boron-doped silicon loaded with hydrogen, measurements of infrared absorption [16] and anelastic relaxation [17] have shown that $\mathrm{H}$ is rather mobile around the substitutional dopant acting as a trapping centre. The measurements of Ref. 17 revealed a thermally activated process due to the $H$ reorientation around $B$ with an activation energy of $W=0.22 \mathrm{eV}$. The pre-exponential factor of the relaxation rate, as derived from the classical Arrhenius law, is $\tau_{0}=1.2 \cdot 10^{13} \mathrm{~s}^{-1}$, and is typical of point defect relaxation. The infrared absorption measurements at low temperature [16] gave relaxation rates slightly higher than the extrapolation from high temperature. The combination of the infrared absorption and of the anelastic relaxation data gives the $\mathrm{H}$ jumping rate in an exceptionally wide range ( 11 orders of magnitude) and the deviation of $\tau^{-1}(T)$ from the classical law displayed at low temperature, suggests the influence of tunnelling. These data have been fitted [18] with the Flynn-Stoneham model [19] of the phonon-assisted incoherent tunnelling for the $\mathrm{H}$ motion.

Direct and unambiguous evidence of quantum tunnelling in semiconductors was recently reported in an anelastic relaxation study of the deuterium dynamics in $\mathrm{GaAs}$ doped with $\mathrm{Zn}$ [20]. A relaxation of $\mathrm{D}$ occurring at $20 \mathrm{~K}$ in the $\mathrm{kHz}$ range has the highest jumping rate found so far for a hydrogen isotope in a semiconductor and cannot be explained in a classical framework.

\section{EXPERIMENTAL PROCEDURE}

The metal samples were two single crystals of $\mathrm{NbZr}_{0.0045}$ electrolytically charged with $\mathrm{H}$ and containing less than 400 at.ppm $\mathrm{O}$ and $\mathrm{N}$. They were two prismatic bars of approximately $2 \times 3 \times 50 \mathrm{~mm}^{3}$; one with the [100] axis and the other with the [111] axis parallel within $3^{\circ}$ to the longer dimension. They were suspended in the central nodal plane and electrostatically excited at one end on their extensional (E) and torsional $(\mathrm{T})$ vibrational modes. We will therefore refer to $\langle 100\rangle \mathrm{E},\langle 100\rangle \mathrm{T},\langle 111\rangle \mathrm{E}$ and $\langle 111\rangle \mathrm{T}$ vibrational modes or deformations. The sample vibration frequency was varied between $3.4 \mathrm{kHz}$ (for flexural vibrations) and $120 \mathrm{kHz}$, and the temperature between $1.1 \mathrm{~K}$ and $300 \mathrm{~K}$. 
The semiconductor samples were four rectangular bars of $40 \times 5 \times 0.4 \mathrm{~mm}^{3}$, as follows: i) $p$-type Si:B with $[\mathrm{B}]=10^{19} \mathrm{~cm}^{-3}$; ii) $n$-type Si:P with $[\mathrm{P}]=5 \times 10^{18} \mathrm{~cm}^{-3}$; ii) $p$-type $\mathrm{GaAs}: \mathrm{Zn} \mathrm{Ga}_{\mathrm{Ga}}$ with $[\mathrm{Zn}]=10^{19}$ $\mathrm{cm}^{-3}$; iv) $n$-type $\mathrm{GaAs}: \mathrm{Si}_{\mathrm{Ga}}$ with $[\mathrm{Si}]=2 \times 10^{18} \mathrm{~cm}^{-3}$. Hydrogen and deuterium were introduced: i) by lowenergy ion-gun irradiation $(\sim 140 \mathrm{eV})$ at $300^{\circ} \mathrm{C}$ for $450 \mathrm{~min}$ for a $\mathrm{H}$ or $\mathrm{D}$ dose of $6.7 \times 10^{18} \mathrm{H}$ ions $\mathrm{cm}^{-2}$; ii) by low pressure plasma ( $0.5 \mathrm{mbar})$ at $300-420^{\circ} \mathrm{C}$ for $180-500 \mathrm{~min}$. In this way, $H(D)$ penetration into the bulk occurs via hopping instead of implantation and the lattice damage is minimized, if not avoided. The acoustic spectroscopy measurements were carried out between $1 \mathrm{~K}$ and $550 \mathrm{~K}$ by electrostatically exciting flexural vibrational modes of the bars which were suspended with thin wires on their nodal lines. The 2nd, 4th, and 6th modes were excited, with resonant frequencies spanning from about $1 \mathrm{kHz}$ to $30 \mathrm{kHz}$.

The temperature was measured by a thin Au- 0.03 at.\% Fe vs chromel thermocouple attached to the suspension point or line with silver paint.

\section{EXPERIMENTAL RESULTS AND DISCUSSION}

\subsection{Metals}

Two types of $\mathrm{H}$ motion are observed in many metals: a fast tunnelling motion between close sites, mainly near a trapping impurity, and a slower hopping around the same impurities and throughout the lattice. The faster motion is associated with the formation of coherent tunnel systems; i.e. $H$ is delocalized over two or more sites and remains in a same eigenstate for a time longer than the tunnelling period. Increasing temperature, the interaction with the phonon and electron bath induces $H$ transitions at a faster rate, until the coherence of the wave function over the different sites is lost and tunnelling becomes incoherent. The slower hopping occurs between different tunnel systems or over longer distances.

\subsubsection{Hopping}

As the solubility of hydrogen in bcc metals at low temperature becomes very small (a few at ppm) and practically all the $\mathrm{H}$ content passes to the precipitated phase, the $\mathrm{H}$ motion cannot be studied in the pure lattice. Interstitial (e.g. $\mathrm{O}, \mathrm{N}, \mathrm{C}$ ) or substitutional (e.g. $\mathrm{Ti}, \mathrm{Zr}$ ) lattice impurities act as trapping centres for $\mathrm{H}$ and keep it in solid solution, allowing the $\mathrm{H}$ dynamics at low temperature to be investigated.

The first evidence of non-classical hopping of $H(D)$ was given in the remote past for the $\mathrm{NbO}_{\mathrm{x}}(\mathrm{H}, \mathrm{D}) \mathrm{y}$ system [21], where a thermally activated energy loss peak at $100 \mathrm{~K}$ (in the $\mathrm{kHz}$ range) was found, with an activation energy of $0.18 \mathrm{eV}$; the process was later ascribed to the relaxation of the $\mathrm{O}-\mathrm{H}$ pair $[22,23]$. In addition, the isotope effect found [21] was very large and not explainable in the classical framework. The peak is not a single Debye process, and this can be understood considering, from the configuration of the $\mathrm{O}-\mathrm{H}$ complex, that in the $\mathrm{H}$ reorientation around $\mathrm{O}$ partial dissociation of the $\mathrm{O}-\mathrm{H}$ complex occurs and different types of jumps are involved.

An extension of the measurements of the relaxation rate down to $40 \mathrm{~K}$ in the same $\mathrm{NbO}_{\mathrm{x}} \mathrm{H}_{\mathrm{y}}$ system revealed [24] a deviation from the classical Arrhenius law, which was interpreted [1] in terms of the small polaron Flynn-Stoneham model of the phonon-assisted incoherent tunnelling [19]. The nonclassical motion of $\mathrm{H}$ can be revealed also at room temperature where, however, also quantum mechanical models tend to the Arrhenius law; in fact, the activation energy for the long range diffusion of free $\mathrm{H}$ in $\mathrm{Nb}$, as derived from the static [25] and dynamic [26] Gorsky effect $(0.10 \mathrm{eV})$, is smaller than the energy of the first vibrational mode [27], and the relaxation time for diffusion presents a strong isotope effect $[21,25,26]$.

Hydrogen hopping, with non classical isotope effect, was later observed in the same temperature range as above also in substitutional solutions like $\mathrm{NbTi}_{\mathbf{x}} \mathrm{H}_{\mathbf{y}}[28,29], \mathrm{VTi}_{\mathbf{x}} \mathrm{H}_{\mathbf{y}}[30]$ and $\mathrm{NbZr}_{\mathbf{x}} \mathrm{H}_{\mathbf{y}}[15,31]$. 


\subsubsection{Coherent Tunnelling}

3.1.2.1. Two-level systems. The most studied coherent tunnel system is that formed by $\mathrm{H}$ near $\mathrm{O}$ in $\mathrm{Nb}$, and has been succesfully interpreted in terms of the TLS model [12]. The corresponding Hamiltonian in the localized representation (i.e. with the two eigenstates representing $\mathrm{H}$ localized in either site) is:

$$
H_{T L S}=\frac{1}{2}\left(\begin{array}{cc}
a & t \\
t & -a
\end{array}\right)
$$

being $t$ the tunnelling matrix element and $a$ the site-energy difference or asymmetry (Fig. 1a); this energy shift $a$ is generally due to the long-range elastic interactions among the O-H pairs. The two energy levels, or eigenvalues of the Hamiltonian are:

$$
E_{1}=-\frac{1}{2} \sqrt{t^{2}+a^{2}}, E_{2}=\frac{1}{2} \sqrt{t^{2}+a^{2}}
$$

and the energy split is:

$$
E_{1,2}=E_{2}-E_{1}=\sqrt{t^{2}+a^{2}} \text {. }
$$

It is generally observed that the effect of deformation is much stronger on the site energies $\pm a / 2$ than on the tunnelling energy $t$. For example, for the TLS of $\mathrm{H}$ in $\mathrm{Nb}$ the energy shifts due to the random strains may be from 0 to tens of kelvin, while the tunnelling energy changes little around its mean value of a few kelvin. For this reason, it is assumed that the strain $\varepsilon$ affects the TLS only through the asymmetry $a=\alpha \varepsilon$, where $\alpha$ is called deformation potential; $\varepsilon$ includes the local strain introduced by the defect interaction and the strain externally applied by the sample vibration. The quantity of interest for the anelastic relaxation is the change of the TLS energy split due to the application of strain which, from Eq. (3), is:

$$
\frac{d E_{12}}{d \varepsilon}=\alpha \frac{a}{E_{12}}
$$

The dependence of the TLS energy levels on strain, i.e. on asymmetry, is shown in Fig. 1c.

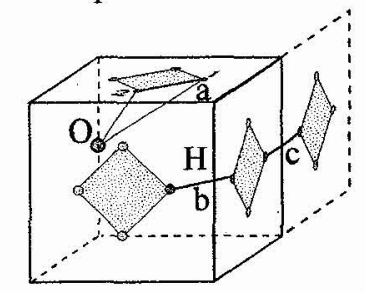

$a / 2$ (1)-(2) $-a / 2$

a)

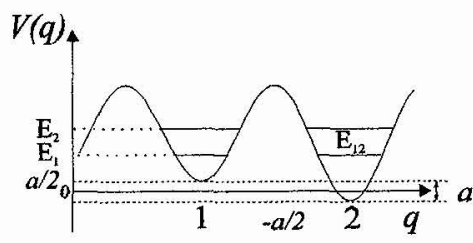

b)

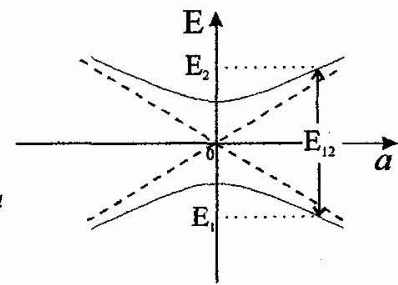

c)

Figure 1: a) Possible pairs of interstitial sites for a TLS of $\mathrm{H}$ near the interstitial $\mathrm{O}$ trapping centre. The two sites "a" have been proposed for the TLS giving rise to peak P1 [5]. The pairs " $b$ " or " $c$ " may be responsible for P2; b) potential energy profile of $\mathrm{H}$ in a TLS; c) energy levels of the TLS as a function of asymmetry $a$.

In the absence of any interaction of the TLS with the electron and/or phonon bath, the particle tunnels coherently between two sites with frequency $t / h$. The transitions between the two energy levels occur because of the interaction of the TLS's with phonons and/or electrons. The resulting relaxation rate 
of the TLS's is $\tau^{-1}=\tau_{12}^{-1}+\tau_{21}^{-1}$, where $\tau_{i j}^{-1}$ is the transition rate from level $i$ to level $j$. The possible interactions inducing the $\mathrm{H}$-transitions in the tunnel systems are the phonon- and electron-interactions. Phonon interactions. The most important types of transitions induced by phonons are the following. One-phonon transitions. They are direct transitions from level $E_{1}$ to level $E_{2}$ (and viceversa) through the absorption (or emission) of one phonon with energy $E_{12}$ (Fig. 2a). The contribution of such transitions to the relaxation rate $\tau_{1 p h}^{-1}$ is:

$$
\tau_{1 p h}^{-1}=\left(\tau_{0}^{-1}\right)_{1 p h} \cdot \operatorname{coth}\left(\frac{E_{12}}{2 k T}\right)
$$

which is a function slowly varying with temperature (in the high temperature approximation $\tau^{-1} \propto T$ ). These transitions take place mainly at the lowest temperatures.

Two-phonon transitions. The transitions from level $E_{1}$ to level $E_{2}$ (and viceversa) occur via a higher energy level (Fig. 2b). The relaxation rate $\tau_{2 p h}^{-1}$ due to this transition is governed by the law:

$$
\boldsymbol{\tau}_{2 p h}^{-1}=\left(\tau_{0}^{-1}\right)_{2 p h} \cdot \cosh \left(\frac{E_{12}}{2 k T}\right) \cdot\left(\frac{E_{12}}{2 k T}\right)^{-7}
$$

which increases very fast with increasing temperature, and is expected to prevail on one-phonon processes above a few kelvin.

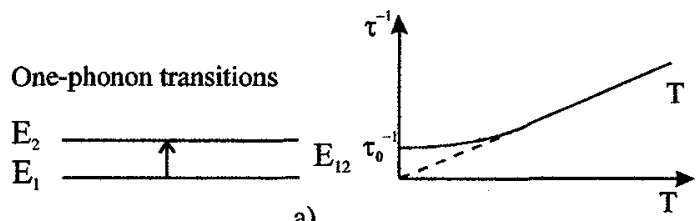

a)

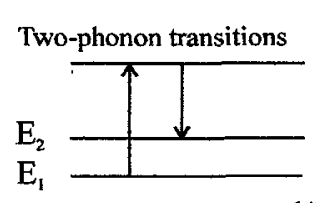

b)

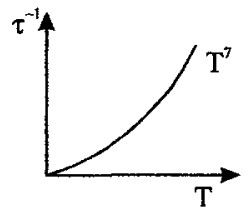

$\mathrm{T}$

Figure 2: Temperature dependence of the contribution to the relaxation rate from one- and two-phonon processes.

Electron interactions. Whilst in insulating materials like glasses or alkali halides only phonon interactions are possible, in metals electron interactions can become predominant in assisting the transitions of the tunnelling systems. Electrons can exert markedly different influences on TLS's, depending on the state of the metal. The coupling of the TLS's with the conduction electrons gives rise to a relaxation frequency which is slowly varying with temperature in the normal state where, according to the simplest model, follows the law [32]:

$$
\tau_{e l}^{-1}=\left(\tau_{0}^{-1}\right)_{e l} \cdot \operatorname{coth}\left(\frac{E_{12}}{2 k T}\right)
$$

More elaborate models predict different temperature dependencies, but always slowly varying. In the superconducting state, the relaxation frequency rapidly decreases with decrease of temperature as [33]:

$$
\tau_{e l}^{-1} \propto k T e^{-\frac{\Delta_{G}}{k T}}
$$


where $\Delta_{G}$ is the superconducting energy gap. This occurs because the number of free electrons available for the interaction with the TLS's is reduced; thus, the tunnelling dynamics in the superconducting state is remarkably slowed when $\mathrm{T}$ is decreased.

The most studied alloy in terms of two-level tunnel system in metals, the $\mathrm{O}-\mathrm{H}$ pair in $\mathrm{Nb}$, has been investigated by the following three types of experiments.

3.1.2.2. Specific heat: Each TLS with energy split $E_{i j}$ contributes to the specific heat at a temperature $k T \approx E_{12}$, and therefore from specific heat measurements it is possible to extract information on the density of states of the tunnel systems. The first indication of $\mathrm{H}$ tunnelling in $\mathrm{Nb}-\mathrm{O}$ came from this type of experiments [2]. The distribution of the values of $E_{i j}$ depends on that of $a$ and has a lower cutoff at $t$ (Eq.1); this is reflected in the specific heat as a low temperature cutoff. The values of $t$ estimated in $\mathrm{Nb}$ $\mathrm{O}$ from the position of this cutoff [3] are $t \approx 0.2 \mathrm{eV}$ for $\mathrm{H}$ and $t \approx 0.02 \mathrm{eV}$ for D.

3.1.2.3. Anelastic relaxation. The elastic energy dissipation due to relaxation between two states with energies $E_{1}$ and $E_{2}$ is:

$$
Q^{-1}(T) \propto\left(\frac{\partial E_{12}}{\partial \varepsilon}\right)^{2} \frac{1}{k T} \operatorname{sech}^{2}\left(\frac{E_{12}}{2 k T}\right) \frac{\omega \tau}{1+(\omega \tau)^{2}},
$$

where the relaxation strength is expressed in terms of the strain derivative of the energy split instead of the change of the elastic dipole; according to Eq. 4, it is proportional to:

$$
\Delta_{12} \propto\left(\frac{\partial E_{12}}{\partial \varepsilon}\right)^{2} \propto \alpha^{2}\left(\frac{a}{E_{12}}\right)^{2} .
$$

The fact that the relaxation strength is proportional to the square of the asymmetry can be intuitively understood by observing that at lower asymmetry $\mathrm{H}$ becomes more uniformly delocalised in both sites, so that the transitions between the two levels involve a smaller rearrangement of the $H$ probability density.

The anelastic relaxation spectrum in the $\mathrm{kHz}[10]$ and $\mathrm{MHz}[7,8]$ range revealed that there are two $\mathrm{H}(\mathrm{D})$ tunnelling peaks in $\mathrm{Nb}-\mathrm{O}-\mathrm{H}$ at low temperature, which are located between 1 and $8 \mathrm{~K}$, depending on frequency and isotope. The peak at lower temperature, called P1, is due to the same TLS's giving rise to the low energy excitations observed by the above mentioned specific heat measurements [3], and to the inelastic peaks observed by neutron scattering [5] to be mentioned below. Other neutron scattering experiments suggested for P1 a TLS within the pair of sites indicated as "a" in Fig. 1a.

The nature of the second process, labelled as P2, is more uncertain and three proposals have been put forward. It was attributed to a TLS [10] originating in the same O-H complex but of a different configuration [34]. Indeed, for a TLS there exist three pairs of interstitial sites symmetrically placed (and hence energetically equivalent) around $\mathrm{O}$ (Fig. 1a). Whatever the microscopic configuration is, $\mathrm{P} 2$ should be due to a TLS, because there are no more than two equivalent and close enough positions for $\mathrm{H}$ near $\mathrm{O}$. The analysis of P2 in terms of the standard TLS model gave the unusual result of a same tunnelling matrix element $t \approx 1 \mathrm{meV}$ for both $\mathrm{H}$ and $\mathrm{D}[10]$, whereas $\mathrm{D}$ is expected to have a tunnelling energy lower than that of $\mathrm{H}$, as found for P1. It was also suggested that $\mathrm{P} 2$ is due to $\mathrm{O}-2 \mathrm{H}$ complexes, on the basis of the dependence of its height on the cooling rate and ageing at different temperatures [35], and finally to a process connected with the motion of dislocations [36,37]. Further experiments are needed to clarify the nature of the relaxation process $\mathrm{P} 2$.

The determination of the interactions assisting the $\mathrm{H}$ transitions between the energy levels was not an obvious task; in fact, the analysis of peak $\mathrm{P} 2$ in $\mathrm{NbO}_{\mathrm{x}} \mathrm{H}_{\mathrm{y}}$ in terms of both electron interactions in the superconducting state and two-phonon interactions, gave similar results [10] (one-phonon interactions would give peaks extremely broad with respect to the experimental curves). This is due to the fact that 
the relaxation rates in the two cases have similar temperature dependences according to Eqs. 6 and 8 . The three following experiments demonstrated that, in general, electron interactions are predominant at low temperatures in bcc metals. In $\mathrm{NbO}_{\mathrm{x}} \mathrm{H}_{\mathrm{y}}$, the authors of Refs. 11 and 38 observed that process $\mathrm{P} 1$ disappears under the application of a magnetic field forcing the sample into the normal state, as is to be expected due to the variation of regime of the relaxation rate from the superconducting to the normal state of a TLS (Eqs. 8 and 7, respectively). In $\mathrm{TaO}_{\mathrm{x}} \mathrm{D}_{\mathrm{y}}$ the discovery [39] of a dissipation effect shaped as a very broad curve from $50 \mathrm{~K}$ to $T_{c}=4.5 \mathrm{~K}$ (the normal to superconducting transition), and as a peaked curve below $T_{c}$, was satisfactorily interpreted [39] in terms of a unique relaxation due to TLS's and changing its $\tau^{-1}$ regime on passing across $T_{c}$ (see Eqs.7 and 8). The three above experiments led to the conviction that, in general, electron interactions are predominant at low temperatures in metals.

3.1.2.4. Neutron spectroscopy. The presence of TLS's gives rise to inelastic peaks centred at $\pm t$ in the intensity of the scattered neutrons, whose shape depends on the distribution of the values of $a$ and $t$ and on the TLS transition rate $\tau^{-1}$ [5]. At temperatures higher than $\sim 10 \mathrm{~K}$ the coherence of the $\mathrm{H}$ tunnelling is increasingly destroyed and the neutron spectra become quasi-elastic; the incoherent hopping rate between the two sites can be deduced from the width of the quasi-elastic peak. It has been possible to evaluate $t$ and the distribution of $a$ as a function of the impurity concentration, which correlate well with the values obtained by the other methods. The measurement of $\tau^{-1}$ has been extended [6] above $5 \mathrm{~K}$ to values of the order of $10^{-10} \mathrm{~s}^{-1}$ : a relevant result is the decrease of the rate between 10 and $60 \mathrm{~K}$, which has been found quantitatively consistent with the recent models of non-adiabatic interaction with the electrons, although the interpretation has been questioned [40].

The tunnelling parameters obtained for $\mathrm{H}$ in $\mathrm{Nb}-\mathrm{O}$ [6] are in excellent agreement with those from the specific heat experiments.

3.1.2.5. Four-level system. The existence of four-level tunnel systems had long been postulated in substitutional alloys for symmetry reasons [34, 13], and is corroborated by elastic energy-loss experiments in $\mathrm{NbZr}_{0,0045}$ single crystals charged with $\mathrm{H}$ [15].

The theory of the FLS is much less developed than that of the TLS, and the dissipation curves have a more complex shape. In Ref. [15] an analysis has been made of the effect of the higher symmetry of the FLS on the response of the tunnel system to external stress, compared to the TLS case. Figure 3a represents the case of tunnelling of $\mathrm{H}$ among the four tetrahedral sites coordinated with a substitutional impurity atom acting as a trapping centre, for instance $\mathrm{Ti}$ or $\mathrm{Zr}$ in $\mathrm{Nb}$. In the first approximation, the site energy shifts of a FLS due to the random internal strains can be taken as centrosymmetric, with pairs of opposite sites shifted by the same amount $a / 2$ as in Fig. 3a, because strain is a centrosymmetric tensor. Instead, a centrosymmetric TLS is necessarily also symmetric and does not relax. The resulting Hamiltonian, with the notation adopted for the TLS, is:

$$
H_{T L S}=\left(\begin{array}{cccc}
a & t & 0 & t \\
t & -a & t & 0 \\
0 & t & a & t \\
t & 0 & t & -a
\end{array}\right) .
$$

The corresponding energy levels (eigenvalues), represented in Fig. 3c, are

$$
E_{1}=-\frac{1}{2} \sqrt{4 t^{2}+a^{2}}, E_{2}=-\frac{a}{2}, E_{3}=\frac{a}{2}, E_{4}=\frac{1}{2} \sqrt{4 t^{2}+a^{2}}
$$

and the eigenfunctions are 


$$
|1,4\rangle=\frac{1}{2 \sqrt{E(E \pm a)}}\left[\begin{array}{c}
2 t \\
-(a \pm E) \\
2 t \\
-(a \pm E)
\end{array}\right], \quad|2\rangle=\frac{1}{\sqrt{2}}\left[\begin{array}{c}
0 \\
1 \\
0 \\
-1
\end{array}\right],|3\rangle=\frac{1}{\sqrt{2}}\left[\begin{array}{c}
1 \\
0 \\
-1 \\
0
\end{array}\right]
$$

and reflect the symmetry of the Hamiltonian. The application of an external strain $\varepsilon$ perturbs the Hamiltonian by $\delta H_{F L S}$; neglecting the change of the tunnelling energies, as above for the TLS, and admitting the most general case in which each site energy changes by $a_{i}=\alpha, \varepsilon, \delta H_{F L S}$ assumes the form:

$$
\delta H_{T L S}=\left(\begin{array}{cccc}
\alpha_{1} & 0 & 0 & 0 \\
0 & \alpha_{2} & 0 & 0 \\
0 & 0 & \alpha_{3} & 0 \\
0 & 0 & 0 & \alpha_{4}
\end{array}\right)
$$

The changes of the energy levels are obtained from the mean values of $\delta H_{F L S}$ over the four eigenstates, $d E_{i} / d \varepsilon=\left\langle i\left|\delta H_{F L S}\right| i>\right.$, and can be written as:

$$
\frac{\partial E_{1}}{\partial \varepsilon}=\bar{\alpha}-\frac{a}{2 E_{14}} \delta, \quad \frac{\partial E_{2}}{\partial \varepsilon}=\bar{\alpha}-\delta, \quad \frac{\partial E_{3}}{\partial \varepsilon}=\bar{\alpha}+\delta, \quad \frac{\partial E_{4}}{\partial \varepsilon}=\bar{\alpha}+\frac{a}{2 E_{14}} \delta,
$$

with:

$$
\bar{\alpha}=\frac{1}{4}\left(\alpha_{1}+\alpha_{2}+\alpha_{3}+\alpha_{4}\right) \text { and } \delta=\frac{1}{4}\left(\alpha_{1}+\alpha_{3}-\alpha_{2}-\alpha_{4}\right)
$$

The resulting intensities of the relaxations between levels $i$ and $j$ are:

$$
\Delta_{i j} \propto\left(\frac{\partial E_{i}}{\partial \varepsilon}-\frac{\partial E_{j}}{\partial \varepsilon}\right)^{2} \propto \delta^{2} \propto\left(\alpha_{1}+\alpha_{3}-\alpha_{2}-\alpha_{4}\right)^{2},
$$

and are all proportional to $\delta^{2}$. The quantity $\delta \varepsilon$ is just the change of the difference between the mean site energies of the two pairs of opposite sites under application of $\varepsilon$, and this result is a consequence of the symmetry of the $\mathrm{H}$ wave function. Instead, for an asymmetric TLS within any pair of $\mathrm{T}$ sites, there is no cancellation of the site energy changes, since the asymmetric wave function weights the two site energy changes differently.

3.1.2.6. The $\mathrm{NbZr}_{x} \mathrm{H}_{y}$ system. The symmetry of the $\mathrm{Zr}-\mathrm{H}$ pair in $\mathrm{Nb}$ containing substitutional $\mathrm{Zr}$ was investigated in detail [15]. Figure 4 shows the energy dissipation curves versus temperature of the two differently oriented $\mathrm{NbZr}_{0.0045} \mathrm{H}_{\mathrm{x}}$ single crystals. The dissipation of the $\mathrm{H}$-free samples is rather low and displays only a small kink at the normal to superconducting transition temperature. The $\angle 100>\mathrm{E}$ and $<111>\mathrm{T}$ modes of the samples charged with $\mathrm{H}$ display a thermally activated peak at about $2 \mathrm{~K}$, whereas the $\langle 100>\mathrm{T}$ (not shown in Fig.4) and $<111>\mathrm{E}$ modes present no relaxation in the same region. The $\mathrm{H}$ contents were $\mathrm{x}=0.0011$ for the $<111>$ and $\mathrm{x}=0.00065$ for the $<100>$ oriented crystals, respectively. For the sake of clearness, the set of experiments of Fig. 4 is schematically represented in Fig. 5 assuming tunnelling of $\mathrm{H}$ among the four tetrahedral sites of a face of the cell containing $\mathrm{Zr}$, as in Fig. 3 . 


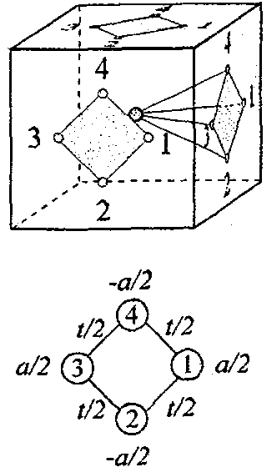

a)
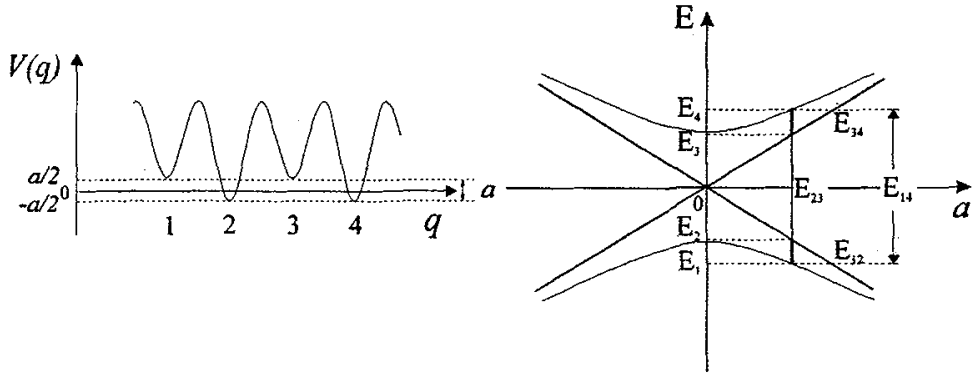

c)

Figure 3: Four-level system; a) interstitial sites for a FLS of $\mathrm{H}$ near the substitutional $\mathrm{Zr}$; $b$ ) potential energy profile of $H$ in the FLS; c) energy levels of the FLS as a function of asymmetry $a$.

If we suppose that the deformation induces a site energy change which is proportional to the variation of the distance of the site from the $\mathrm{Zr}$ atom, then in the case of $\angle 100>\mathrm{E}$ vibration we have for the FLS evidenced in the figure, $\alpha_{1}=\alpha_{3}=\alpha, \alpha_{2}=\alpha_{4}=-\alpha$ in Eq. (16), and the relaxation strength is proportional to the square of $\delta=\alpha$. The $<111>\mathrm{T}$ vibration distorts the unit cell in a completely nonsymmetric way, resulting in a finite $\delta$. Instead, in the case of $\angle 111>E$ and $\angle 100>T$ vibrations, it is $\alpha_{1}=\alpha_{4}, \alpha_{2}=\alpha_{3}$ and $\alpha_{1}=\alpha_{3}=0, \alpha_{2}=-\alpha_{4}$, respectively. In both cases it is $\delta=0$, and therefore there is no relaxation, as experimentally observed.

The experimental results of Fig. 4, follow the behaviour predicted for a nearly centrosymmetric FLS, and not for a TLS, and therefore they constitute an indication of the existence of four-level systems in metallic substitutional solutions.
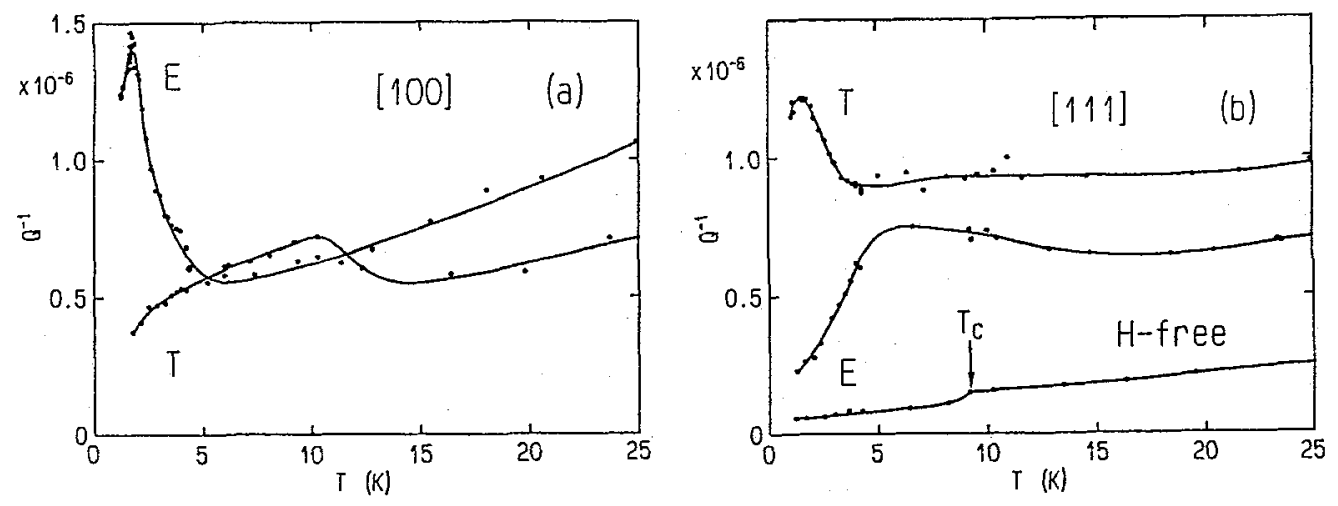

Figure 4: Elastic energy loss attributed to coherent tunnelling of $\mathrm{H}$ neat $\mathrm{Zr}$ in $\langle 100\rangle$ oriented $\mathrm{NbZr}_{0.0045} \mathrm{H}_{0.00065}$ and $\langle 111\rangle$ oriented $\mathrm{NbZr}_{0.0045} \mathrm{H}_{0.0011}$ single crystals. The vibration frequencies were $42 \mathrm{kHz}(<100>\mathrm{E}), 46 \mathrm{kHz}(<100>\mathrm{T}), 28 \mathrm{kHz}(<111>\mathrm{E})$ and $52 \mathrm{kHz}(<111>\mathrm{T})$.

The indication that FLS's exist in bcc substitutional systems also comes from specific heat measurements in $\mathrm{NbTi}_{0.05}(\mathrm{H}, \mathrm{D})_{\mathrm{y}}$ [14]; they display an excess specific heat which remains remarkably 
high down to $50 \mathrm{mK}$, whereas in $\mathrm{NbO}_{\mathbf{x}}(\mathrm{H}, \mathrm{D})_{\mathrm{y}}$ it drops [3] when $k T<t$ which is the minimum energy split of the TLS's. A difference in the shape and values of the specific heat curves is expected from the two types of multi-level systems. In fact, with increase of the asymmetry $a$, the separation of the first two levels of a TLS increases (Eq. 3 and Fig. 1c), whilst that of an FLS approaches zero as $t^{2} / a^{2}$ (Fig. 3c). Thus, the low temperature specific heat in the substitutional alloys would be due to the large strains introduced by the high Ti concentration, and not to a tunnelling matrix element much smaller than those found in $\mathrm{NbO}_{\mathrm{x}} \mathrm{H}_{\mathrm{y}}$.
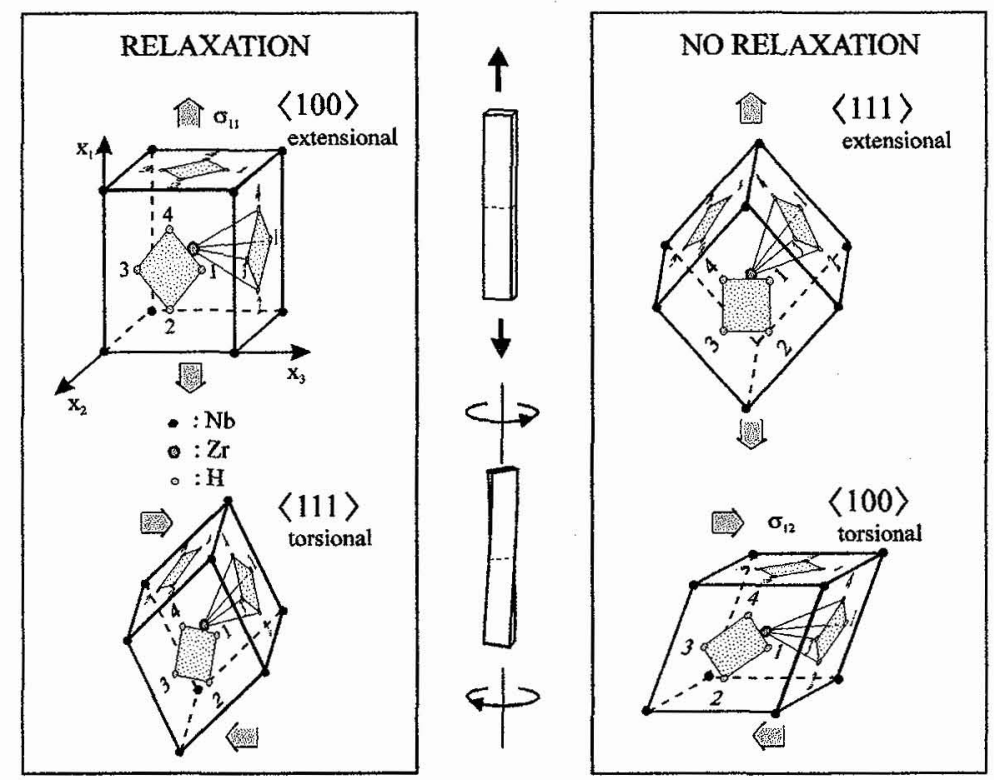

Figure 5: Representation of the distortions of the $\mathrm{Nb}$ cubic cell in the experiments of Fig. 4.

\subsection{3. $Y H_{x}$.}

Hydrogen can go in solid solution in great quantities also in the hcp rare earths and form relatively stable $\mathrm{H}$ pairs which prevent the formation of hydride phases at concentrations $\mathrm{H} / \mathrm{Y}$ as high as $0.2-0.3$. The occupation is mainly tetrahedral, and the $T$ sites are in close pairs along the $c$ axis, which can be occupied by no more than one $\mathrm{H}$ atom; the thermodynamically stable $\mathrm{H}$ pairs are formed within two pairs of $\mathrm{T}$ sites separated by a metal atom. Tunnelling within the close pairs of $\mathrm{T}$ sites seems the only possibility, because the other inter-site distances are definitely higher; nonetheless, $\mathrm{H}$ relaxation involving tunnelling occurs over at least two time scales. Very fast transition rates (over $10^{10} \mathrm{~s}^{-1}$ ) have been observed by quasi-elastic neutron scattering [41], with characteristics similar to those of the $\mathrm{O}-\mathrm{H}$ TLS in Nb; NMR [42] and ultrasonic absorption experiments [43] reveal a definitely slower relaxation, while from lower frequency anelastic experiments even lower relaxation rates are found [44]. A possible explanation for these observations is that in all the cases the TLS's are within the same type of close T sites, but with widely different asymmetries, depending whether the $\mathrm{H}$ atom is isolated, or belongs to a $\mathrm{H}$ pair, or is close to an O impurity [44]. Since the relaxation rates of the TLS's are proportional to $(t / E)^{2}$, the higher is the asymmetry and the lower is the relaxation rate. 


\subsection{Semiconductors}

\subsubsection{The Si:B and Si:P Systems.}

Figure 6 shows the elastic energy dissipation curve of Si:B as a function of temperature. The sample in the $\mathrm{H}$-free state displays a monotonical decrease of dissipation with decreasing temperature. After hydrogenation, a well developed peak appears above the background at about $125 \mathrm{~K}$ (at $2.4 \mathrm{kHz}$ ). The main features of that peak are the following. The peak shifts in temperature with frequency, indicating a thermally activated nature, and is described by a single-time Debye curve. In the temperature range of the energy-loss measurements, the relaxation time is well fitted by an Arrhenius law ( $\tau=\left[(8 \pm 4) 10^{-14} \mathrm{~s}\right] e^{(0.22 \pm 0.01) \mathrm{eV} / k r}$ ). The peak is stable (the cooling curves are retraced on heating), with a height increase depending on the $\mathrm{H}$ content, whilst the maximum temperature is concentration independent; the elastic interactions among the $\mathrm{B}-\mathrm{H}$ complexes do not appear to affect the $\mathrm{H}$ jumps within the experimental uncertainty, and an upper limit of less than $10 \mathrm{meV}$ is estimated for the random shifts of the $\mathrm{H}$ site energies due to such interactions. As hydrogen and its isotopes are expected to have a bond-centre occupancy in p-type doped Si (nearly half-way in the bond between the substitutional dopant and the host atom, see Fig. 6), this peak has been attributed to the stress-induced reorientation of $H$ among the four bond-centre sites around $B$ [17]. The single-time nature of the relaxation process is consistent with the high dilution of the B-H complexes $\left(\sim 10^{19} \mathrm{~B}\right.$ atoms $\left./ \mathrm{cm}^{-3}\right)$.

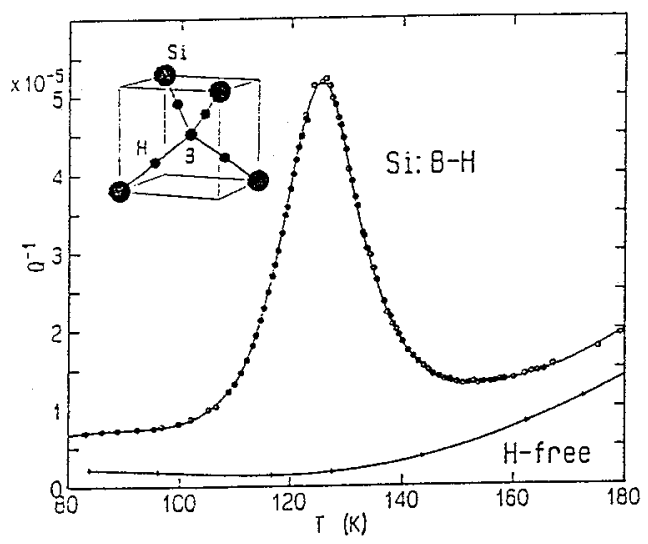

Figure 6: Elastic energy dissipation coefficient versus temperature of $\mathrm{H}$-free and hydrogenated $\mathrm{Si}: \mathrm{B}$. The (thermally activated) relaxation peak is due to the reorientation of $\mathrm{H}$ around $\mathrm{B}$. The sketch shows the four equivalent bond-centre positions of $\mathrm{H}(\mathrm{D})$ in $\mathrm{Si}: \mathrm{B}$.

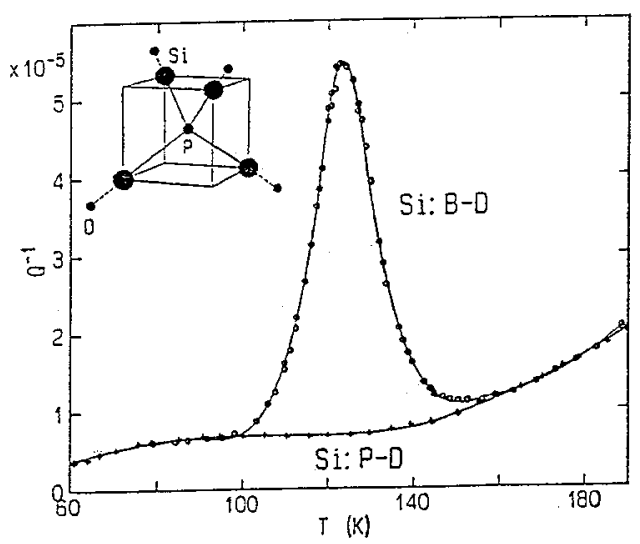

Figure 7: Elastic energy dissipation coefficient versus temperature of $p$-type Si:B-D displaying the peak due to the B-D relaxation, and of $n$-type Si:P-D showing the absence of any process (in the range $1 \mathrm{~K}-550 \mathrm{~K}$; only a limited range is shown in the figure). The sketch shows the backbonding positions of H(D) in Si:P.

Hydrogen in $n$-type semiconductors is expected to occupy sites different from the bond-centre positions generally expected in $p$-type semiconductors. To test the different $\mathrm{H}$ occupancy, we carried out acoustic spectroscopy measurements in $n$-type, Si:P charged with $\mathrm{H}$ and $\mathrm{D}$. In this system, $\mathrm{H}$ is expected to occupy a backbonding site, i.e. the site on the P-Si bond line, back to the Si atom such that Si bridges the $\mathrm{H}$ and $\mathrm{P}$ atoms, as shown in Fig. 7. This figure also shows the dissipation curves versus $\mathrm{T}$ of deuterated Si:P and of Si:B as a reference. Whilst the peak due to the stress-induced reorientation of $D$ among the bond-centre sites around $B$ appears in Si:B-D, no process has been observed in Si:P-D in the same temperature range. It is surprising that even in the extended range $1-550 \mathrm{~K}$, no relaxation process 
could be detected in Si:P. As the backbonding sites are far apart, it is conceivable that the H(D) transitions among the four energetically equivalent positions cannot occur via direct jumps, but require partial dissociation of the complex. In this case the relaxation strenght is expected to be strongly reduced and the absence of relaxation processes in Si:P supports the backbonding occupancy for $H(D)$ in $n$-type doped Si.

\subsubsection{The GaAs:Zn and GaAs:Si systems.}

The coefficient $Q^{-1}$ of the elastic energy dissipation of deuterated GaAs: $\mathrm{Zn}$ at two different frequencies (1 and $13 \mathrm{kHz}$ ) is plotted in Fig. 8. A well developed peak appears at about $20 \mathrm{~K}$ which shifts with frequency thus indicating that the process is thermally activated. The peak is governed by unexpectedly high $\mathrm{H}$ transition rates, more than 15-20 orders of magnitude higher than in all the other semiconductors measured so far. Moreover, the peak is much broader than a single Debye process. In the same Fig. 8 the dissipation curves of deuterium-free GaAs:Zn and of D-loaded GaAs:Si are also plotted. In all cases the peak is absent, indicating that the presence of both $\mathrm{Zn}$ and $\mathrm{D}$ is necessary for its appearance. Although the above observations suggest that the peak is caused by the relaxation of $\mathrm{D}$ around the $\mathrm{Zn}$ trapping centre, an attribution to a particular complex symmetry and mechanism is not possible at present.

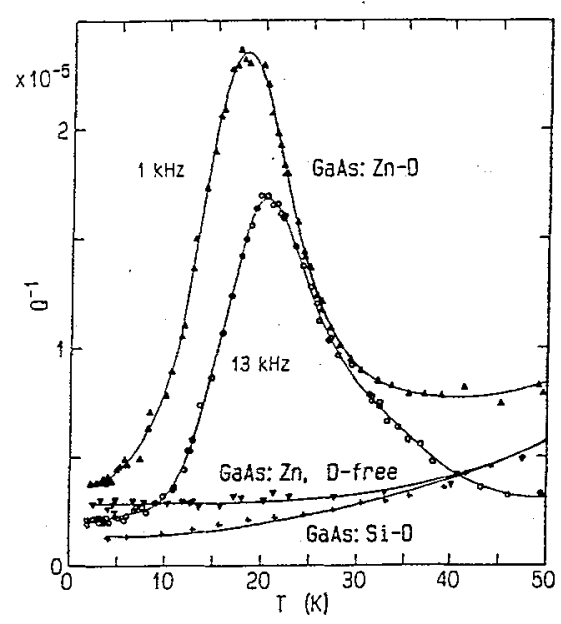

Figure 8 Elastic energy dissipation coefficient versus temperature of $p$-type GaAs: $\mathrm{Zn}-\mathrm{D}$ at 1 and $13 \mathrm{kHz}$ showing the thermally activated $\mathrm{Zn}-\mathrm{D}$ peak around $20 \mathrm{~K}$, of D-free GaAs: $\mathrm{Zn}$, and of $n$-type GaAs:Si-D displaying only background dissipation.

The above $\mathrm{Zn}-\mathrm{D}$ peak cannot be described by classical hopping. In fact, the single-time Debye curve, traced by using the Arrhenius law for $\tau(T)$ and the activation energy $W$ obtained from the peak shift with frequency, is far too narrow with respect to the experimental curve. The curve broadening can be explained in terms of a distribution of the $D$ site energies, i.e. of both the activation energies $W$ and site asymmetries $a$ caused by the elastic interactions among the $\mathrm{Zn}-\mathrm{D}$ complexes. However these interactions have shown to be negligible in Si:B-H at the low dopant concentrations used $\left(\sim 10^{19} \mathrm{~cm}^{-1}\right)$, where the B-H relaxation gives rise to a single Debye process [17]. In an attempt to introduce large distributions of $W$ and $\tau_{0}^{-1}(T)$ into the classical formulae, $\tau_{0}^{-1}(T)$ had to be varied of several orders of magnitude in order to obtain a reasonable fit to the experimental curves, and thus it assumed completely unphysical values.

Having provided enough evidence that the $D$ dynamics in GaAs is markedly quantistic, two possibilities have been considered, the incoherent and the coherent $\mathrm{D}$ tunnelling. A widely used model for incoherent tunnelling is the model of Flynn and Stoneham [19], in which the $\mathrm{H}$ atom is supposed to be self-trapped in a given site and to tunnel to an adjacent site when the two site energies are made equivalent by thermal fluctuations. A tentative fit in terms of this model was accomplished [20] and the result was only apparently satisfactory, because of the large variation introduced for the tunnelling matrix 
element $J$ in order to fit the data. Although the values assumed for $J$ are not as unphysical as they are for $\tau_{0}^{-1}(T)$, they are rather high for a tunnelling process. Moreover, the distribution for $J$ is too broad to be caused by elastic interactions, at least at the low $\mathrm{Zn}$ and $\mathrm{D}$ concentrations presently used.

Coherent tunnelling has been reported in H(D)-metal systems. In the present case, H(D) can be shifted by the large and heavy substitutional $\mathrm{Zn}$ to three equivalent off-axis positions around the $\mathrm{Zn}$-As bond. The confinement of $H(D)$ in these sites should give rise to three-level tunnel systems at low temperature and the H(D) transitions among quantized energy levels could then be the origin of the observed relaxation. Also the tunnelling among the sites of the four different bonds around $\mathrm{Zn}$ cannot be excluded. An analysys of the dynamic regime for $\mathrm{H}$ or $\mathrm{D}$ in GaAs: $\mathrm{Zn}$ is in progress.

Lastly, the absence of any peak (Fig. 8) over the range $1-550 \mathrm{~K}$ in the $n$-type GaAs:Si-D system, where $\mathrm{H}$ and its isotopes are expected to occupy antibonding sites (on the $\mathrm{Si}$-As bond line, back to the $\mathrm{Si}$ atom such that $\mathrm{Si}$ bridges the $\mathrm{H}$ and $\mathrm{As}$ atoms) may indicate that the height of the energy barrier separating the four equivalent antibonding sites around $\mathrm{Si}$ is fairly high.

\section{CONCLUSION}

The dynamics of interstitial $\mathrm{H}$ in bcc and fcc metals is dominated by tunnelling, even in the case of the slower quasi-classical hopping. At lower temperature, the very fast motion between close sites near trapping impurities becomes coherent tunnelling. Some remarkable success has been achieved in the interpretation of the experimental results in terms of the TLS model, and many indications have also been obtained that $\mathrm{H}$ may be coherently delocalised over four sites in the symmetrical environment of a substitutional impurity in bcc metals. Nonetheless, still much work has to be done, especially on the bcc substitutional alloys and the hcp rare earths.

The relaxation of H-B pairs in Si gives rise to a nearly single Debye process. The relaxation rates from the anelastic relaxation or from the decay of dichroism, considered separately, are well fitted by a classical Arrhenius law. The combination of data from the two types of experiments reveals a deviation from the exponential behaviour at low temperature. A preliminary attempt in terms of a small polaron model of the phonon-assisted incoherent tunnelling has been accomplished. The absence of relaxation effects in the Si:P-D system is consistent with the backbonding occupancy of $\mathrm{H}(\mathrm{D})$ in $n$-type doped Si. A new peak observed in GaAs:Zn-D at $20 \mathrm{~K}$, much broader than a single Debye curve, is caused by a very mobile species identifyed as the $\mathrm{Zn}-\mathrm{D}$ complex. The mechanism causing the process cannot be explained classically, and at present both the under-barrier $\mathrm{H}$ hopping and the $\mathrm{H}$ coherent tunnelling between definite lattice sites should be considered. Finally, the deuterated $n$-type GaAs:Si system, where H(D) is expected to occupy $\mathrm{Si}$ antibonding sites, does not display relaxation processes, thus indicating a rather high activation energy for the reorientation of $\mathrm{D}$ around $\mathrm{Si}$ in $\mathrm{GaAs}$.

\section{References}

[1] P.E. Zapp and H.K. Bimbaum, Acta Metall. 28, 1523 (1980).

[2] G.J. Sellers, A.C. Anderson and H.K. Birnbaum, Phys. Rev. B 10, 2771 (1974).

[3] C. Morkel, H. Wipf and K. Neumaier, Phys. Rev. Lett. 40, 947 (1978); H. Wipf and K. Neumaier, ibid., 52, 1308 (1984).

[4] H. Wipf, A. Magerl, S.M. Shapiro, S.K. Satija and W. Thomlinson, Phys. Rev. Lett. 46, 947 (1981).

[5] A. Magerl, A.J. Dianoux, H. Wipf, K. Neumaier and I.S. Anderson, Phys. Rev. Lett. 56, 159 (1986).

[6] D. Steinbinder, H. Wipf, A.J. Dianoux, A. Magerl, K. Neumaier, D. Richter and R. Hempelmann, Europhys. Lett. 16, 211 (1991).

[7] D.B. Poker, G.C. Setser, A.V. Granato and H.K. Birnbaum, Z. Phys. Chem. 116, 39 (1979).

[8] D.B. Poker, G.C. Setser, A.V. Granato and H.K. Birnbaum, Phys. Rev. B 29, 622 (1984).

[9] G. Cannelli and R. Cantelli, Solid State Comm. 43, 567 (1982). 
[10] G. Cannelli, R. Cantelli and F. Cordero, Phys. Rev. B 34, 7721 (1986).

[11] W. Morr, A. Müller, G. Weiss, H. Wipf and B. Golding, Phys. Rev. Lett. 63, 2084 (1989).

[12] J. Jäckle, L. Piché, W. Arnold and S. Hunklinger, J. Non-Crystalline Solids 20, 365 (1976).

[13] G. Cannelli and R. Cantelli, F. Cordero and F. Trequattrini, Z. Phys. Chem. 179, 317 (1993); G. Cannelli, R. Cantelli and G. Vertechi, Appl. Phys. Lett. 39, 832 (1981).

[14] K. Neumaier, H. Wipf, G. Cannelli and R. Cantelli, Phys. Rev. Lett. 49, 1423 (1982).

[15] G. Cannelli, R. Cantelli, F. Cordero and F. Trequattrini, Phys. Rev. B 49, 15040 (1994).

[16] M. Stavola, K. Bergman, S.J. Pearton, and J. Lopata, Phys. Rev. Lett. 61, 2786 (1988).

[17] G. Cannelli, R. Cantelli, M. Capizzi, C. Coluzza, F. Cordero, A. Frova, and A. Lo Presti, Phys. Rev. B 44, 11486 (1991).

[18] Y.M. Cheng and M. Stavola, Phys. Rev. Lett. 73, 3419 (1994).

[19] C.P. Flynn and A.M Stoneham, Phys. Rev B 1, 3966 (1970).

[20] G. Cannelli, R. Cantelli, F. Cordero, E. Giovine, F. Trequattrini, M. Capizzi, and A. Frova, Solid State Comm. 98, 873 (1996).

[21] G. Cannelli and L. Verdini, Ric. Sci. 36, 98 (1966); G. Cannelli and L. Verdini, Ric. Sci. 36, 246 (1966).

[22] C.C. Baker and H.K. Bimbaum, Acta Metall. 21, 865 (1973).

[23] R.F. Mattas and H.K. Birnbaum, Acta Metall. 23, 973 (1975).

[24] C.G. Chen and H.K. Birnbaum, Phy. Stat. Sol. (A) 36, 687 (1976).

[25] G. Schaumann, J. Völkl and G. Alefeld, Phys. Rev. Lett. 21, 891 (1968).

[26] R. Cantelli, F.M. Mazzolai and M. Nuovo, Phys. Stat. Sol. 34, 597 (1969).

[27] A. Magerl, J.J. Rush and J.M. Rowe, Phys. Rev. B 33, 2093 (1986).

[28] G. Cannelli and R. Cantelli, Proc. of the 6th International Conference on Internal Friction and Ultrasonic Attenuation in Solids, ICIFUAS-6, Tokyo 1977, edited by R.R. Hasiguti and N. Mikoshiba (University of Tokyo Press), p.491, 1979.

[29] G. Cannelli, R. Cantelli and M. Koiwa, Phil. Mag. A 46, 483 (1982).

[30] S. Tanaka and M. Koiwa, Scripta Metall. 15, 403 (1981).

[31] G. Cannelli and R. Cantelli, F. Cordero and F. Trequattrini, J. Alloys Comp. 211/212, 80 (1994).

[32] J.L. Black, in: Glassy Metals I, Springer Topics in Applied Physics, Vol. 46 (Springer, Berlin, 1981); Amorphous Solids, ed. by W.A. Phillips, Springer Topics in Applied Physics (Springer, Berlin, 1981).

[33] J.L. Black and P. Fulde, Phys. Rev. Lett. 43, 453 (1979).

[34] G. Cannelli, R. Cantelli and F. Cordero, Z. Phys. Chem. Neue Folge 164, 943 (1989).

[35] K.F. Huang, A.V. Granato and H.K. Birnbaum, Phys. Rev. B 32, 2178 (1985).

[36] E.J. Kramer and C.L. Bauer, Phys. Rev. 163, 407 (1967).

[37] P.P. Pal Val, V.D. Natsik and L.N. Pal Val, Low Temp. Phys. 21, 505 (1995).

[38] E. Drescher-Krasicka and A.V. Granato, J. de Physique C10-46, 73 (1985).

[39] G. Cannelli, R. Cantelli and F. Cordero, Phys. Rev. B 35, 7264 (1987).

[40] I. Svare, Phys. Rev. B 40, 11585 (1989).

[41] I.S. Anderson, A. Heidemann, J.E. Bonnet, D.K. Ross, S.K.P. Wilson, and McKergow, J. LessCommon Met. 101, 405 (1984).

[42] I. Svare, D.R. Torgeson, and F. Borsa, Phys. Rev. B 43, 7448 (1991).

[43] R.G. Leisure, R.B. Schwarz, A. Migliori, D.R. Torgeson, I. Svare, and I.S. Anderson, Phys. Rev. B 48, 887 (1993).

[44] G. Cannelli, R. Cantelli, F. Cordero, F. Trequattrini, I.S. Anderson, J.J. Rush, Phys. Rev. Lett. 67, 2682 (1991); G. Cannelli, R. Cantelli, F. Cordero, F. Trequattrini, and A. Sermoneta, 11th Int. Conf. on Internal Friction and Ultrasonic Attenuation in Solids, ICIFUAS 11, Poitiers 1996. 